\title{
ESTRUTURA DE PROPRIEDADE E MITIGAÇÃO DAS EMISSÕES DE GASES DO EFEITO ESTUFA
}

\author{
Victor Daniel Vasconcelos \\ Mestrando em Administração e Controladoria \\ Universidade Federal do Ceará Fortaleza - CE - Brasil \\ victordanielvasconcelos@alu.ufc.br https://orcid.org/0000-0002-1756-6241 \\ Mônica Cavalcanti Sá de Abreu \\ Doutorado em Engenharia de Produção \\ Universidade Federal do Ceará Fortaleza - CE - Brasil \\ mabreu@ufc.br http://orcid.org/0000-0001-9408-5604 \\ Vicente Lima Crisóstomo \\ Doutor em Nuevas Tendencias En Dirección de Empresas \\ Universidade Federal do Ceará Fortaleza - CE - Brasil \\ vlc@ufc.br http://orcid.org/0000-0002-8593-0471

\section{Robson Silva Soe Rocha} \\ Doutor em Administração Internacional \\ Aarhus University Aarhus C - Dinamarca \\ rocha@mgmt.au.dk http://orcid.org/0000-0002-4539-036X
}

\section{RESUMO}

Esse trabalho investiga o impacto da estrutura de propriedade nas decisões que afetam a mitigação de emissões de gases do efeito estufa (GEE). Esta pesquisa tem como base a teoria da agência e assume a hipótese de que a adoção de estratégias climáticas corporativas é influenciada pela estrutura de propriedade da empresa. Foram analisadas empresas brasileiras de capital aberto que realizaram seu inventário de emissão de GEE entre os anos de 2010 e 2018. Realizou-se análise descritiva e foram processadas estimações por dados em painel de efeitos aleatórios e, como variável dependente, utilizou-se o logaritmo natural da razão entre emissões de GEE e receita bruta. Resultados econométricos indicam que um maior percentual de ações nas mãos de investidores institucionais favorece a redução de emissões de GEE. No caso de propriedade de ações da empresa nas mãos de diretores executivos e de investidores ativistas, não foi identificada uma relação significante com a redução de emissões de GEE. A pesquisa reforça a importância de tipos de estrutura de propriedade em decisões que proporcionam incentivos para enfrentar o aquecimento global.

Palavras-chave: Estratégias. Estrutura de propriedade. Mudanças climáticas. Teoria da agência.

\section{OWNERSHIP STRUCTURE EFFECT ON GREENHOUSE GAS EMISSIONS MITIGATION DECISIONS}

\begin{abstract}
This paper aims to investigate the impact of ownership structure on greenhouse gas (GHG) emissions mitigation decisions. This research is based on the agency theory and assumes that the adoption of corporate climate strategies is influenced by the ownership structure. We analyzed publicly traded Brazilian companies that carried out their GHG emission inventory between 2010 and 2018. Descriptive analysis was performed and estimates were processed by panel data random effects and as dependent variable was used the natural logarithm of the ratio between GHG emissions and gross revenue. Econometric results indicate that there is an insignificant relationship between executive ownership and GHG emissions. Institutional ownership has a negative and significant relationship with GHG emissions and activist investors has a insignificant relationship with GHG emissions. The research reinforces the importance of ownership structure which provides incentives to face global warming.
\end{abstract}

Key words: Agency theory. Climate change. Ownership structure. Strategy.

Data da submissão: $17 / 12 / 2020$

Data de aceite: $23 / 05 / 2021$

Revista de Gestão Social e Ambiental - RGSA, São Paulo, v. 14, n. 3, p. 93-109, set./dez. 2020. 
Estrutura de propriedade e mitigação das emissões de gases do efeito estufa

\section{INTRODUÇÃO}

A emissão de gases do efeito estufa (GEE) (e.g. $\mathrm{CO}_{2}$, metano, óxido nitroso e clorofluorcarbono) aumenta a quantidade de energia que é mantida na atmosfera em decorrência da absorção do calor refletido ou emitido pela superfície do planeta e provoca a elevação da temperatura da atmosfera (Braga et al., 2005). O aquecimento global pode provocar mudanças climáticas, além de outros efeitos, tais como acidificação e elevação do nível dos oceanos, extinção de espécies e escassez nos recursos hídricos (Howard-Grenville et al., 2014). A preocupação de distintos atores sociais com os efeitos do aquecimento global tem gerado pressões sobre empresas para que tenham um comportamento adequado ao risco climático. Estudos empíricos foram realizados para descrever fatores com potencial para impulsionarem as empresas para responderem às mudanças climáticas (Cadez \& Czerny, 2016; Eleftheriadis \& Anagnostopoulou, 2017; Gasbarro et al., 2017; Goldstein et al., 2019; Talbot \& Boiral, 2015). Devido às particularidades na forma como as empresas funcionam e como seriam afetadas pelas mudanças climáticas, não há consenso sobre quais os fatores que supostamente impulsionam (ou não) a definição de estratégias para mitigação de emissões de GEE.

O Brasil, entre economias emergentes, foi um dos primeiros países a assumir compromissos climáticos, sob o Acordo de Paris. Contudo, no ano de 2019, o Brasil lançou 2,17 bilhões de toneladas brutas de dióxido de carbono equivalente ( $\mathrm{tCO} 2 \mathrm{e})$ na atmosfera, o que representa um aumento de $9,6 \%$ em comparação ao ano de 2018 , quando o país lançou 1,98 bilhão, consolidando a reversão da tendência de redução de emissões no Brasil, observada entre os anos de 2004 e 2010 (Observatório do Clima, 2020). A partir do ano de 2019, observa-se o aprofundamento de medidas adotadas para enfraquecer a gestão ambiental pública e eliminar a regulamentação existente (Adebayo et al., 2021), tais como a tentativa de liberar a exploração de petróleo em áreas sensíveis e extinção do Instituto Chico Mendes (Observatório do Clima, 2021). Desta forma, as projeções de emissões de GEE no país foram revisadas para cima, refletindo a tendência crescente da taxa de desmatamento (United Nations Environment Programme, 2019), o Brasil ficou 180\% acima da meta que foi traçada na Política Nacional de Mudanças Climáticas de limitar o desmatamento na Amazônia em 2020 para, no máximo, 3925 km² (Observatório do Clima, 2021).

Dada a incerteza dos efeitos das mudanças climáticas, bem como suas soluções para mitigação e adaptação, pressões de partes interessadas podem influenciar as empresas a desenvolverem estratégias climáticas (Abreu et al., 2021). Ativistas associados a movimentos de combate ao aquecimento global pressionam governos a estabelecerem restrições sobre emissões de GEE, o que pode encorajar empresas a tomarem medidas para ampliarem a divulgação de informações sobre os planos de mitigação (Reid e Toffel, 2009). Essas pressões, acompanhadas pelo maior conhecimento da sociedade, podem influenciar investidores na escolha do seu portfólio de investimentos (Damert \& Baumgartner, 2018), e demandar transparência sobre emissões corporativas de GEE e as estratégias adotadas para reduzi-las (Lee et al., 2015).

As empresas mobilizam-se em diferentes tipos de estratégicas climáticas (Cadez et al., 2019; Hossain \& Farooque, 2019) com o objetivo de ter um equilíbrio entre legitimidade e competitividade (Bui \& Fowler, 2019), as quais demandam capacidades e recursos financeiros, tecnológicos e humanos (Lopes de Sousa Jabbour et al., 2020). Os elementos estratégicos a serem considerados, incluem aumento da eficiência operacional, modificação dos processos de produção e desenvolvimento de produtos menos intensivos em emissões de GEE. Outros desafios incluem o desenvolvimento de abordagens de comunicação eficazes com as partes interessadas para apoiarem os esforços corporativos na redução de emissões de GEE, a participação em processos políticos e a exploração de novas oportunidades de mercado.

A estrutura de propriedade pode impactar nas decisões de investimento na implementação de estratégias climáticas. Elgergeni et al., (2018), Chen et al. (2020) e Martínez-Ferrero e Lozano (2021) trazem evidências de uma relação positiva entre um maior percentual de ações nas mãos de investidores institucionais e a melhoria no desempenho ambiental. Cundill et al. (2018), Michelon

Revista de Gestão Social e Ambiental - RGSA, São Paulo, v. 14, n. 3, p. 93-109, set./dez. 2020. 
et al. (2020) e Qi et al. (2020) indicam que um maior percentual de ações nas mãos de investidores ativistas permite um maior comprometimento com aspectos ambientais significativos. Por outro lado, Cai et al. (2011) sinalizam não ter influência um maior percentual de ações nas mãos de diretores executivos em atividades ambientais.

Este trabalho amplia o debate acerca do efeito da estrutura de propriedade na estratégia climática, ao examinar a seguinte questão de pesquisa: Em que extensão a estrutura de propriedade de empresas em operação no Brasil influencia a tomada de decisões que reduzem emissões de $G E E$ ? A pesquisa parte do princípio que a estrutura de propriedade é um atributo que pode ter efeito sobre as políticas corporativas, incluindo aquelas relacionadas com decisões sobre a mitigação de emissões de GEE As mudanças climáticas demandam respostas cooperativas e integradas, que, por sua vez, exigem planejamento, coordenação e mobilização de conhecimento (Jamieson \& Jacquet, 2020) e, neste sentido, o estudo contribui ao analisar fatores associados à estrutura de propriedade que influenciam (ou não) a tomada de decisões relacionadas com a gestão de emissões de GEE.

O trabalho está estruturado em seções. O referencial teórico, aborda a relação entre teoria da agência e estratégias climáticas, e fundamenta as hipóteses da pesquisa que tratam do efeito da estrutura de propriedade em decisões que reduzem emissões de GEE. Na metodologia, foi analisada a estrutura de propriedade de 52 empresas não financeiras, compondo um total de 321 observações no período 2010-2018. Modelos econométricos foram estimados usando dados em painel com efeitos aleatórios, tendo como variável dependente a eficiência de emissões de GEE, representado pelo "Índice de Carbono Eficiente (ICO2)" e, como variáveis explicativas, os percentuais de ações nas mãos de diretores executivos, de investidores institucionais, e de investidores ativistas. Os resultados da modelagem econométrica são discutidos e refletem a influência da estrutura de propriedade na adoção de estratégias climáticas.

\section{TEORIA DA AGÊNCIA E ESTRATÉGIAS CLIMÁTICAS CORPORATIVAS}

A teoria da agência pode ser utilizada para entender a influência de determinados tipos de estrutura de propriedade da empresa no desempenho ambiental corporativo. Essa teoria baseia-se na relação entre principais (acionistas) e agentes (gestores), onde os agentes agem para atender aos interesses dos principais. O principal usa ferramentas para monitorar qualquer comportamento oportunista do agente com a implementação de incentivos para reduzir à divergência de interesses (Vitolla et al., 2020). Contudo, a separação da propriedade e o controle de uma empresa podem apresentar problemas (Kumala \& Siregar, 2020). Com relação a decisões que envolvem estratégias climáticas, os agentes podem implementar práticas sociais e ambientais para disfarçarem um comportamento oportunista e obterem apoio de partes interessadas, quando são questionados pelos principais (Endo, 2020).

A visão da gestão da empresa, como também de seus proprietários, acerca de questões ambientais é, portanto, importante nas decisões que envolvem a gestão de emissões de GEE. Esta visão pode contribuir para harmonizar a necessidade do comportamento ambientalmente correto com os interesses de investidores. $O$ investimento em ações ambientais tem, via de regra, custo elevado, o que vem a ser um fator desestimulante para sua implementação frente aos objetivos de desempenho financeiro (Badrul et al., 2018). A literatura tem argumentado, e documentado algumas evidências da participação de ações nas mãos de diretores executivos, investidores institucionais ou investidores ativistas podem ter visões distintas sobre as prioridades definidas na política ambiental (Cundill et al., 2018; Malik et al., 2020; Oikonomou et al., 2020).

\subsection{Influência de ações nas mãos dos diretores executivos em decisões que afetam a mitigação de emissões de GEE}

A propriedade em mãos de diretores da empresa leva em conta sua contribuição para o alinhamento de interesses entre propriedade e gestão, quando o gestor também é proprietário, existe 
a possibilidade de "entrincheiramento" se esta propriedade interna for muito elevada (Hadlock, 1998). A propriedade de ações nas mãos dos diretores executivos permanece uma questão de interesse em finanças corporativas (Malik et al., 2020). Diretores executivos são responsáveis pelas decisões mais importantes das empresas (Jaroenjitrkam \& Zurbruegg, 2020) e são os principais tomadores de decisões de uma empresa e os representantes desta externamente (Ji et al., 2019).

Existe uma argumentação dupla sobre uma maior porcentagem de ações nas mãos de diretores executivos e aspectos ambientais. Por um lado, a perspectiva de gestão voltada para o resultado no curto prazo tende a desestimular investimentos de custo elevado com retorno incerto. Um acionista, mesmo na condição de executivo da empresa, tende a ter foco no desempenho em detrimento de atividades "aparentemente" não rentáveis de elevado custo. O poder da direção pode priorizar desempenho em detrimento de investimentos elevados de retorno incerto, que não sejam compulsórios, e omitir informação detalhada a respeito de decisões desta natureza (Malik et al., 2020). Nesta mesma linha, há argumentação no sentido de que o nível de preocupação com atividades ambientais é menor se o poder de tomada de decisões estiver concentrado nas mãos de diretores executivos (Ji et al., 2019). Diretores executivos com muito poder tendem a estar mais focados em interesses associados à sua atividade de direção que visa maximização de resultado em seu período de gestão o que influencia, desfavoravelmente, as decisões corporativas em relação às atividades socioambientais, que podem representar investimento de retorno incerto (Badrul et al., 2018).

Por outro lado, conflitos de agência são atenuados, uma vez que a propriedade da empresa nas mãos dos diretores executivos contribui para o alinhamento de interesses entre propriedade e gestão (Sakawa \& Watanabel, 2019). Grandes acionistas tendem a ter uma perspectiva de mais longo prazo na propriedade da empresa, o que pode suscitar o interesse no investimento, mesmo de alto vulto, que possa contribuir para a minimização do envolvimento da empresa em sinistros de qualquer ordem e, assim, melhorar a imagem e reputação da empresa. Sob esta perspectiva, investimentos socioambientais tendem a gerar retorno no longo prazo, o que é mais importante para grandes acionistas com expectativa de manutenção de posição na propriedade da empresa no longo prazo do que para acionistas minoritários. Uma elevada proporção de ações nas mãos de diretores executivos pode fazer com que estes se unam a acionistas que queiram prosperidade da empresa no longo prazo (Kim \& Kim, 2015).

No entanto, sendo a proporção de propriedade executiva pouco relevante, como é o caso no mercado brasileiro, não se tem expectativa de que o comportamento executivo venha a alinhar-se fortemente com a visão de longo prazo. Pesquisas não têm encontrado relação entre propriedade executiva e ações ambientais da empresa (Cai et al., 2011). Diante da não existência de um consenso teórico e empírico da relação entre propriedade nas mãos de diretores executivos e desempenho ambiental, observa-se que o objetivo de curto prazo da direção executiva da empresa é prevalente em mercados nos quais esta propriedade é pouca elevada, como é o caso do mercado brasileiro. Assim, considera-se a seguinte hipótese:

\section{Hipótese 1: Propriedade da empresa nas mãos dos diretores executivos não favorece decisões que reduzem emissões de GEE.}

\subsection{Influência de ações nas mãos de investidores institucionais em decisões que afetam a mitigação de emissões de GEE}

A chamada propriedade institucional refere-se aos investimentos de investidores institucionais, como fundos de pensão, seguradores e fundos mútuos (Oh et al., 2011). Esta característica da estrutura de propriedade da empresa, i.e., a propriedade institucional, tem sido bem vista pelo mercado. Investidores institucionais têm sido considerados como capazes de atenuar conflitos de agência envolvendo gestores e acionistas, considerando sua perspectiva de investimento de longo prazo e seu envolvimento na monitoração da gestão que contribui para o maior 
alinhamento de interesses entre propriedade e gestão (Oh et al., 2017). Empresas com acionistas institucionais têm apresentado tendência de geração de melhores resultados de impacto social e ambiental, devido ao atendimento de demandas de partes interessadas e a redução de riscos, além da perspectiva de longo prazo destes investidores (Chen et al., 2020).

Pelo espectro de investimentos socialmente responsáveis, a propriedade de empresas em mãos de investidores institucionais tem-se tornado cada vez mais importante, com fundos de investimento sendo estruturados para atender pressão advinda de partes interessadas (Oikonomou et al., 2020). A perspectiva de investimento de longo prazo dos investidores institucionais, tem sido vista como capaz de permitir um maior nível de engajamento em atividades sociais e ambientais, uma vez que este envolvimento é positivo para o desempenho de longo prazo da empresa e para sua imagem e reputação (Elgergeni et al., 2018).

Neste contexto, a questão da legitimação das atividades corporativas também pode ser um fator importante no sentido de motivar investidores institucionais ao empreendimento de ações socioambientais (Ullah et al., 2019). Evidência prévia sinaliza que, de fato, a propriedade da empresa em mãos de investidores institucionais parece ser favorável ao empreendimento de estratégias climáticas (Martínez-Ferrero e Lozano 2021; Chen et al. 2020; Elgergeni et al., 2018). Esta argumentação e evidência anterior motivam a proposição da hipótese que sugere que a propriedade da empresa brasileira por investidores institucionais é benéfica à mitigação de emissões de GEE, como a seguir expressa:

\section{Hipótese 2: Propriedade de ações nas mãos de investidores institucionais favorece decisões que reduzem emissões de GEE.}

\subsection{Influência de ações nas mãos de investidores ativistas em decisões que afetam a mitigação de emissões de GEE}

O ativismo dos acionistas é um processo pelo qual os acionistas buscam influenciar as ações da empresa a seu favor, inibindo inclinações dos gestores a priorizarem seus próprios interesses (Monks, 2008). Neste sentido, o ativismo dos acionistas pode ser uma forma de movimento social para evitar o excesso de poder de diretores executivos das empresas (Hadani et al., 2019). O ativismo dos acionistas é uma forma de atuação para estes proporem mudanças nas empresas, pelo levantamento de questões que expõem suas opiniões (Wei, 2020). Sob a perspectiva da teoria da agência, o ativismo dos acionistas pode ser tido como um mecanismo de governança corporativa ao ser um instrumento de controle da atividade da direção da empresa, atuando na diminuição dos conflitos de agência (Goranova et al., 2017).

O ativismo dos acionistas surge, quando estes acreditam que a administração da empresa falhou em seu dever, ou seja, existe uma insatisfação dos acionistas com o desempenho da corporação (Uysal \& Tsetsura, 2015). Investidores ativistas são usualmente acionistas minoritários que se unem para ter poder de pressão sobre a direção da empresa. Nos dias atuais, muitos fundos de hedge, que concentram seus recursos em um portfólio de empresas com ajuda da alavancagem de participações significativas nas empresas, têm assumido uma postura ativista (Jahnke, 2019). Também mais recentemente, acionistas tornaram-se cada vez mais ativos na tentativa de influenciar as práticas sociais e ambientais das empresas (Cundill et al., 2018).

Nesse cenário, investidores que seguem o ativismo dos acionistas podem incentivar as empresas a se envolverem em comportamentos socialmente responsáveis (Cundill et al., 2018; Qi et al., 2020). Este movimento indica uma evolução no ativismo de acionistas que passa a visar não somente os interesses de curto prazo de acionistas minoritários como também estão exigindo uma mais contundente postura socioambiental da empresa. De fato, tem sido detectada evidência de ativismo de acionistas no sentido de impulsionar comportamento ambiental mais intenso da empresa (Michelon et al., 2020; Qi et al., 2020). Este argumento, juntamente com a evidência prévia, motivou a proposição de que propriedade nas mãos de acionistas ativistas é capaz de 
Estrutura de propriedade e mitigação das emissões de gases do efeito estufa

encorajar os gestores a terem atitudes mais benéficas ao meio ambiente, como em relação à redução de emissões de GEE, como a seguir explicitada:

\section{Hipótese 3: Propriedade da empresa nas mãos de investidores ativistas favorece decisões que reduzem emissões de GEE.}

\section{METODOLOGIA}

A pesquisa apresenta abordagem quantitativa, exploratória e descritiva. A população do estudo compreende empresas não financeiras listadas na Bolsa de Valores Brasil, Bolsa Balcão (B $\left.{ }^{3}\right)$ que realizaram seu inventário de emissões de GEE entre os anos de 2010 e 2018, cujas informações foram coletadas nos relatórios anuais ou de sustentabilidade. Empresas financeiras foram removidas por possuírem critérios e regras especiais de operações que podem afetar a divulgação de informações ambientais (Fuente et al., 2017) e as variáveis de controle são influenciadas por práticas contábeis utilizadas por empresas financeiras (Frías-Aceituno et al., 2013). A amostra final do estudo compreende 52 empresas com 321 observações, dispostas em um painel desbalanceado no período de 2010 a 2018. Destaca-se que as estimativas dispostas em painéis desbalanceados são tão confiáveis quanto aquelas baseadas em painéis balanceados (Arellano, 2003). A Tabela 1 apresenta a distribuição setorial da amostra, de acordo com os setores definidos pela $\mathrm{B}^{3}$.

Tabela 1 - Distribuição setorial da amostra

\begin{tabular}{lccc}
\hline \multirow{2}{*}{ Setor } & \multirow{2}{*}{ Empresas } & \multicolumn{2}{c}{ Frequência } \\
\cline { 3 - 4 } & 9 & 59 & Relativa \\
\hline Bens Industriais & 3 & 18 & 18,38 \\
Comunicações & 7 & 34 & 5,61 \\
Consumo cíclico & 8 & 53 & 10,59 \\
Consumo não cíclico & 6 & 39 & 16,51 \\
Materiais básicos & 2 & 6 & 12,15 \\
Petróleo e gás & 2 & 4 & 1,87 \\
Saúde & 15 & 108 & 1,25 \\
Utilidade pública & 52 & 321 & 33,64 \\
\hline Total & & & 100 \\
\hline
\end{tabular}

Fonte: Dados disponíveis na $\mathrm{B}^{3}$

Nota-se, em relação aos setores, maior representatividade do setor de utilidade pública com 15 empresas e 108 observações anuais, seguido pelos setores de consumo não cíclico com 8 empresas e 53 observações anuais, bens industriais com 9 empresas e 59 observações anuais e consumo cíclico com 7 empresas e 34 observações anuais. Os setores de menor representatividade são o de comunicações com 3 empresas e 18 observações anuais, petróleo e gás com 2 empresas e 6 observações anuais e o de saúde com 2 empresas e 4 observações anuais.

\subsection{Definição das variáveis dependentes, independentes e de controle}

A variável dependente do estudo é representada pelo "Índice de Carbono Eficiente (ICO2)", constituído pela razão entre emissões de GEE do escopo 1 (emissões diretas), medido em toneladas de $\mathrm{CO} 2$ equivalente - tCO2 e a receita bruta da empresa em milhões de reais, medindo, assim, a eficiência da gestão de emissões de GEE. Nota-se que o ICO2 tem uma relação inversa, ou seja, quanto menor o seu valor mais eficiente é a empresa na gestão de emissões de GEE (Bui et al., 2020; Qian \& Schaltegger, 2017). O ICO2 possibilita avaliar o desempenho ambiental das empresas e otimizar operações com baixa emissão de GEE (Hua et al., 2016). As informações acerca de emissões de GEE (escopo 1) foram coletadas a partir dos relatórios de sustentabilidade ou anuais das empresas da amostra, e a receita bruta foi obtida na base da Capital IQ.

Revista de Gestão Social e Ambiental - RGSA, São Paulo, v. 14, n. 3, p. 93-109, set./dez. 2020. 
As variáveis independentes envolvem as características da estrutura de propriedade considerada relacionada a três categorias de acionistas: diretores executivos, investidores institucionais e investidores ativistas. A concentração de propriedade nas mãos dos diretores executivos (Dexec) corresponde ao percentual de ações nas mãos de diretores executivos, calculado pela razão entre ações nas mãos de diretores executivos e o total de ações da empresa em determinado ano (Cai et al., 2011; Khan et al., 2013). A concentração de propriedade de investidores institucionais (Inst) é obtida pela razão entre ações nas mãos de investidores institucionais e a quantidade total de ações da empresa em um determinado ano (Martínez-Ferrero e Lozano 2021; Chen et al. 2020). A concentração de propriedade de investidores ativistas (Ativ) é a razão entre o número de ações nas mãos de investidores ativistas e a quantidade de ações da empresa em um determinado ano (Autore et al., 2019; Shi et al., 2019). Estes dados foram obtidos na base Capital IQ.

Variáveis adicionais de controle compõem o modelo de regressão para diminuir a probabilidade de viés nos resultados. A variável IDADE da empresa, medida pelo logaritmo natural da idade da empresa, foi introduzida sob o argumento que empresas mais maduras são mais eficientes na redução das emissões de GEE (Harjoto \& Rossi, 2019). A variável oportunidades de crescimento (Ocresc) é mensurada pelo q de Tobin, que consiste na razão entre a soma do valor de mercado e o passivo exigível sobre ativo total (Zou et al., 2019). Ao tentar maximizar suas oportunidades de crescimento a empresa pode estar sacrificando sua política de mitigação das emissões de GEE.

A variável rentabilidade (ROA) é medida pela relação entre o Ebitda e o ativo total (Michelon et al., 2020). Empresas mais rentáveis teriam mais recursos para investir na gestão de emissões de GEE. O endividamento (END), medido pela relação entre dívida total e ativo total, pode agir como inibidor de uma melhor política de emissão de GEE, face aos compromissos financeiros da empresa (Jackson et al., 2020). O tamanho da empresa (TAM), medido pelo logaritmo do ativo total (Chen et al., 2020) pode ser considerado como favorável a política de emissão de GEE, uma vez que empresas maiores teriam mais recursos e conhecimento acumulado relacionado com projetos de mitigação de emissões de GEE. Os dados referentes as variáveis de controle, foram coletados na base Capital IQ.

\subsection{Estimação dos modelos econométricos e análise dos dados}

O modelo econométrico (apresentado na equação 1) sugere que a eficiência de emissões de GEE é influenciada pela concentração de propriedade nas mãos de diretores executivos, investidores acionistas e investidores ativistas da empresa. Ressalta-se que, devido à presença de outliers, optou-se por transformar o ICO2 na variável Eegee por meio da aplicação de logaritmo natural, que permite a linearização dos dados e a redução dos efeitos de outliers, ademais, como todas as empresas apresentaram emissões de GEE e receita bruta acima de zero, não existe restrição matemática para aplicação do logaritmo natural.

$$
\begin{aligned}
& \text { EEGEE }_{\mathrm{i}, t}=\beta_{0}+\beta_{1} \text { DEXEC }_{i, t}+\beta_{2} \operatorname{INST}_{\mathrm{i}, \mathrm{t}}+\beta_{3} \text { ATIV }_{\mathrm{i}, \mathrm{t}}+\beta_{4} \text { IDADE }_{\mathrm{i}, \mathrm{t}}+\beta_{5} \text { OCRESC }_{i, t}+\beta_{6} \text { ROA }_{i, t}+ \\
& \beta_{7} \text { END }_{i, t}+\beta_{8} \text { TAM }_{i, t}+\varepsilon
\end{aligned}
$$

Tendo como base as variáveis elencadas, foram estabelecidos o modelo 1, somente com as variáveis de controle, os modelos 2,3 e 4, que incluíram as variáveis de controle e as variáveis independentes Dexec, Inst e Ativ, respectivamente, e o modelo 5, que foi gerado com todas as variáveis do estudo. Todos os modelos atendem as hipóteses do estudo. Nesta pesquisa, optou-se pelo modelo de regressão de dados em painel, no qual foram coletadas informações para os mesmos indivíduos ao longo dos anos de 2010 e 2018. A decisão entre os painéis de efeitos fixos, aleatórios ou pooled OLS, se deu por meio dos testes de Hausman, Breush-Pagan e teste F para efeitos

Revista de Gestão Social e Ambiental - RGSA, São Paulo, v. 14, n. 3, p. 93-109, set./dez. 2020. 
Estrutura de propriedade e mitigação das emissões de gases do efeito estufa

individuais. Todas as análises foram executadas com o programa Stata 16.0. Antes da análise de regressão, foi feita uma análise descritiva dos dados, na qual foram extraídas medidas de tendência e variabilidade, para que se pudesse ter uma ideia prévia de como os dados se comportam.

\section{RESULTADOS}

A Tabela 2 apresenta a estatística descritiva das variáveis do estudo. Observa-se que Eegee (logaritmo natural de emissões de gases do efeito estufa pela receita bruta) apresenta uma média de $-4,250$, com valor de $-11,181$, representando a máxima eficiência energética de uma empresa em determinado ano, e valor de $-0,065$, que constitui a mínima eficiência energética de uma empresa para determinado ano.

Tabela 2 - Dados estatísticos descritivos

\begin{tabular}{lccccc}
\hline Variável & Observações & Média & Desvio-Padrão & Mínimo & Máximo \\
\hline EEGEE & 321 & $-4,250$ & 2,495 & $-11,181$ & $-0,654$ \\
DEXEC & 321 & 0,118 & 0,945 & 0 & 8,14 \\
INST & 321 & 27,917 & 20,990 & 0 & 91,87 \\
ATIV & 321 & 0,125 & 0,642 & 0 & 7,22 \\
IDADE & 321 & 3,233 & 0,772 & 1,386 & 4,663 \\
OCRESC & 321 & 1,391 & 0,746 & 0,626 & 6,987 \\
ROA & 321 & 0,079 & 0,059 & $-0,100$ & 1,434 \\
END & 321 & 0,664 & 0,170 & 0,266 & 1,434 \\
TAM & 321 & 16,661 & 1,204 & 13,950 & 20,572 \\
\hline
\end{tabular}

Fonte: Elaborada com base nos dados da pesquisa

Nota-se que a média de ações nas mãos de investidores institucionais $(27,917)$ é bem superior àquela proporção de ações nas mãos de investidores ativistas $(0,125)$ e nas mãos de diretores executivos $(0,118)$. O maior percentual de ações nas mãos de investidores institucionais é de 91,87, enquanto a propriedade de diretores executivos o valor máximo é de 8,14 e de investidores ativistas é de 7,22. Observa-se um maior desvio-padrão em ações de investidores institucionais $(20,990)$, seguido por ações em posse de diretores executivos $(0,945)$ e em propriedade de investidores ativistas $(0,642)$.

\subsection{Análise multivariada}

Dados em painel aprimoram a consistência e o poder explicativo da análise da regressão, fornecendo dados mais informativos com maior variabilidade (García-Sánchez, 2020). Os testes F, Breusch-Pagan Langrange e Hausman foram realizados com o objetivo de analisar o melhor modelo de dados em painel disponível (efeitos fixos, efeitos aleatórios e pooling). O teste $\mathrm{F}(21,72$; $\mathrm{p}=0,000$ ) foi executado indicando que o modelo de efeitos fixos é mais apropriado que o pooling. $\mathrm{O}$ modelo de efeitos aleatórios mostrou ser superior ao pooling, após realização do teste multiplicador de Breusch-Pagan Langrange $(38,1 ; \mathrm{p}=0.000)$. Além disso, ao estudar as mesmas empresas em diferentes períodos de tempo, o modelo pooling se torna automaticamente inadequado (Wooldridge, 2009).

O teste de Hausman $(6,30 ; p=0,6140)$ determina que o modelo de efeitos aleatórios é mais favorável que o de efeitos fixos para o estudo, ao não rejeitar a hipótese nula. Desta forma, adota-se o modelo de dados em painel de efeitos aleatórios. Ressalta-se que o modelo de dados em painel de efeitos aleatórios é considerado o estimador mais eficiente, fornecendo melhores valores-p, devendo ser executado se for estatisticamente justificável (Giovanni \& Mauro, 2019). Ademais, o maior VIF da equação 1 foi de 2,11, com VIF médio de 1,38. Como o valor do VIF não é maior que 10, o estudo não apresenta o problema de multicolinearidade (Kennedy, 2003). A Tabela 3 apresenta os resultados do estudo. 
Victor D. Vasconcelos, Mônica C. Sá de Abreu, Vicente Lima Crisóstomo, Robson Silva Soe Rocha

Tabela 3 - Regressão de dados em painel para a eficiência da gestão de emissões de GEE

\begin{tabular}{|c|c|c|c|c|c|}
\hline \multirow{3}{*}{ Variáveis } & \multicolumn{5}{|c|}{ Dados em painel de efeitos aleatórios } \\
\hline & \multicolumn{5}{|c|}{ Variável dependente: $\ln ($ Emissões de GEE/Receita Bruta) } \\
\hline & Modelo 1 & Modelo 2 & Modelo 3 & Modelo 4 & Modelo 5 \\
\hline & Coef Valor $p$ & Coef Valor $p$ & Coef Valor $p$ & Coef Valor $p$ & Coef Valor $\mathrm{p}$ \\
\hline DEXEC & & $-0,371 \quad 0,171$ & & & $-0,407 \quad 0,133$ \\
\hline INST & & & $-0,0200,001 * * *$ & & $-0,0200,011 * *$ \\
\hline ATIV & & & & $-0,0930,437$ & $-0,050 \quad 0,673$ \\
\hline IDADE & $0,7540,028 * *$ & $0,7450,029 * *$ & $0,7860,021 * *$ & $0,6800,050 * *$ & $0,7170,037 * *$ \\
\hline OCRESC & $0,1160,535$ & $0,0920,621$ & $0,1190,519$ & $0,1210,519$ & $0,100 \quad 0,592$ \\
\hline ROA & $\begin{array}{lll}-2,947 & 0,108\end{array}$ & $-2,7940,128$ & $-2,650 \quad 0,155$ & $-2,901 \quad 0,117$ & $-2,471 \quad 0,179$ \\
\hline END & $-0,255 \quad 0,729$ & $-0,1740,813$ & $-0,040 \quad 0,956$ & $-0,1920,795$ & $0,1020,891$ \\
\hline TAM & $0,058 \quad 0,755$ & $0,027 \quad 0,885$ & $0,059 \quad 0,750$ & $0,088 \quad 0,644$ & $0,048 \quad 0,763$ \\
\hline Constante & $-7,4730,000 * * *$ & $-6,898 \quad 0,000 * * *$ & $-7,191 \quad 0,000 * * *$ & $-7,810 \quad 0,000 * * *$ & $-6,830 \quad 0,000 * * *$ \\
\hline Observações & 321 & 321 & 321 & 321 & 321 \\
\hline Empresas & 52 & 52 & 52 & 52 & 52 \\
\hline Wald Chi2 & $(5) 9,40 * * *$ & (6) $11,34 * * *$ & (6) $16,26 * * *$ & (6) $9,52 * * *$ & (8) $18,06 * * *$ \\
\hline$R$-squared & & & & & \\
\hline Within & 0,024 & 0,045 & 0,0432 & 0,0256 & 0,0458 \\
\hline Between & 0,061 & 0,1193 & 0,0954 & 0,0605 & 0,1193 \\
\hline Overall & 0,059 & 0,1132 & 0,0833 & 0,0631 & 0,1132 \\
\hline Período & 10 & 10 & 10 & 10 & 10 \\
\hline Teste F & $\mathrm{F}=20,11 \quad 0,000$ & $\mathrm{~F}=22,52 \quad 0,000$ & $\mathrm{~F}=23,36 \quad 0,000$ & $\mathrm{~F}=23,12 \quad 0,000$ & $\mathrm{~F}=21,72 \quad 0,000$ \\
\hline Teste de & & & & & \\
\hline $\begin{array}{l}\text { Breusch- } \\
\text { Pagan }\end{array}$ & $\mathrm{LM}=30,40,000$ & $\mathrm{LM}=31,40,000$ & $\mathrm{LM}=37,20,000$ & $\mathrm{LM}=34,10,000$ & $\mathrm{LM}=38,10,000$ \\
\hline $\begin{array}{l}\text { Teste de } \\
\text { Hausman }\end{array}$ & $\mathrm{H}=5,90 \quad 0,3159$ & $\mathrm{H}=6,75 \quad 0,3444$ & $\mathrm{H}=5,79 \quad 0,4468$ & $\mathrm{H}=5,84 \quad 0,4414$ & $\mathrm{H}=6,30 \quad 0,6140$ \\
\hline
\end{tabular}

Fonte: Elaborada com base nos dados da pesquisa

Níveis de significância: $* * * p<0.01 ; *$ p $<0.05 ; * \mathrm{p}<0.1$.

O modelo 1 apresenta uma relação positiva e significante com Idade das empresas, o restante das variáveis de controle não apresenta significância. Nota-se, nos modelos 2 e 5 , uma relação não significante entre um maior percentual de ações nas mãos de diretores executivos e a eficiência na redução de emissões de GEE (Eegee), medida pelo logaritmo da razão entre emissão de GEE e receita bruta. Observa-se, também, nos modelos 3 e 5, que um maior percentual de ações nas mãos de investidores institucionais tem uma relação negativa e significante com Eegee e, consequentemente, ocasionando uma maior eficiência na redução das emissões de GEE. Ademais, percebe-se uma relação não significante, nos modelos 4 e 5, entre um maior percentual de ações nas mãos de investidores ativistas e a variável Eegee. Destaca-se, em todos os modelos, uma relação positiva e significante entre a idade das empresas e Eegee, ou seja, empresas mais antigas tendem a tomar decisões que não favorecem a redução de emissões de GEE. Ressalta-se também relações não significante entre oportunidades de crescimento, rentabilidade, endividamento e o tamanho das empresas com a Eegee.

\subsection{Análise de sensibilidade}

Emissões de GEE, geralmente, são sensíveis ao tamanho da empresa, ou seja, empresas maiores tendem a gerar mais emissões de GEE do que empresas menores (Qian et al., 2018). Desta forma, ajustar a quantidade de emissões pelo tamanho da empresa é uma forma de medir a eficiência de emissões de GEE, sendo utilizada por estudos anteriores (Prasad \& Mishra, 2017; Qian et al., 2018; Trinks et al., 2017). A Tabela 4 apresenta os resultados da análise sensitiva. 
Estrutura de propriedade e mitigação das emissões de gases do efeito estufa

Tabela 4 - Regressão de dados em painel para a eficiência da gestão de emissões de GEE ajustados ao tamanho da empresa

\begin{tabular}{|c|c|c|c|c|c|}
\hline \multirow{3}{*}{ Variáveis } & \multicolumn{5}{|c|}{ Dados em painel de efeitos aleatórios } \\
\hline & \multicolumn{5}{|c|}{ Variável Dependente: $\ln ($ Emissões de GEE/Ativo Total) } \\
\hline & Modelo 1 & Modelo 2 & Modelo 3 & Modelo 4 & Modelo 5 \\
\hline & Coef Valor $\mathrm{p}$ & Coef Valor $\mathrm{p}$ & Coef Valor $\mathrm{p}$ & Coef Valor $\mathrm{p}$ & Coef Valor $\mathrm{p}$ \\
\hline DEXEC & & $-0,2560,371$ & & & $-0,2950,264$ \\
\hline INST & & & $-0,0200,009 * * *$ & & $-0,0200,010^{* *}$ \\
\hline ATIV & & & & $-0,0820,506$ & $-0,0370,762$ \\
\hline IDADE & $0,8090,015 * *$ & $0,8020,016^{* *}$ & $0,8380,012 * *$ & $0,7430,028 * *$ & $0,7830,020 * *$ \\
\hline OCRESC & $0,1550,415$ & $0,1390,466$ & $0,1610,392$ & $0,1590,404$ & $0,1470,436$ \\
\hline ROA & $-1,6170,386$ & $-1,5120,419$ & $-1,3360,470$ & $-1,5830,402$ & $-1,2140,516$ \\
\hline END & $0,1130,879$ & $0,174 \quad 0,815$ & $0,3210,664$ & $0,1730,817$ & $0,440 \quad 0,556$ \\
\hline TAM & $-0,0690,706$ & $-0,0940,614$ & $-0,0660,717$ & $-0,043 \quad 0,818$ & $-0,0760,685$ \\
\hline Constante & $-6,5460,000 * * *$ & $-6,8980,000 * * *$ & $-6,2830,000 * * *$ & $-6,8590,000 * * *$ & $-5,9900,000 * * *$ \\
\hline Observações & 321 & 321 & 321 & 321 & 321 \\
\hline Empresas & 52 & 52 & 52 & 52 & 52 \\
\hline Wald Chi2 & $(5) 7,74 * * *$ & $(6) 8,68 * * *$ & (6) $14,76 * * *$ & $(6) 7,63 * * *$ & (8) 15,31 \\
\hline$R$-squared & & & & & \\
\hline Within & 0,022 & 0,0248 & 0,0432 & 0,0223 & 0,0450 \\
\hline Between & 0,0450 & 0,0538 & 0,0737 & 0,0426 & 0,0795 \\
\hline Overall & 0,0450 & 0,0554 & 0,0634 & 0,0474 & 0,0756 \\
\hline Período & 10 & 10 & 10 & 10 & 10 \\
\hline Teste F & $\mathrm{F}=20,94 \quad 0,000$ & $\mathrm{~F}=20,42 \quad 0,000$ & $\mathrm{~F}=20,95 \quad 0,000$ & $\mathrm{~F}=20,36 \quad 0,000$ & $\mathrm{~F}=19,90 \quad 0,000$ \\
\hline Teste de & & & & & \\
\hline $\begin{array}{l}\text { Breusch- } \\
\text { Pagan }\end{array}$ & $\mathrm{LM}=31,30,000$ & $\mathrm{LM}=33,40,000$ & $\mathrm{LM}=38,30,000$ & $\mathrm{LM}=35,30,000$ & $\mathrm{LM}=39,2 \quad 0,000$ \\
\hline $\begin{array}{l}\text { Teste de } \\
\text { Hausman }\end{array}$ & $\mathrm{H}=8,62 \quad 0,1252$ & $\mathrm{H}=9,29 \quad 0,1579$ & $H=8,57 \quad 0,1993$ & $H=9,71 \quad 0,1375$ & $\mathrm{H}=9,74 \quad 0,2841$ \\
\hline
\end{tabular}

Fonte: Elaborada com base nos dados da pesquisa

Níveis de significância: ${ }^{* *} \mathrm{p}<0.01 ; * \mathrm{*}<<0.05 ;{ }^{*} \mathrm{p}<0.1$.

Notam-se que resultados da tabela 4 são semelhantes aos apresentados na tabela 3 , ou seja, para o modelo 1, a idade apresenta uma relação positiva e significante, os modelos 2 e 5 mostram uma relação não significante entre um maior percentual de ações nas mãos de diretores executivos e a eficiência de emissões de GEE (Eegee). Observa-se, nos modelos 3 e 5, uma relação negativa e significativa entre um maior percentual de ações nas mãos de investidores institucionais e a eficiência de emissões de GEE, realçando que a propriedade institucional favorece decisões que reduzem emissões de GEE. Os modelos 4 e 5 confirmam que não existe influência de um maior percentual nas mãos de investidores ativistas na variável Eegee. Além disso, ressalta-se, em todos os modelos, a relação positiva e significante entre idade das empresas e eficiência de emissões de GEE e a relação não significante entre oportunidade de crescimento, rentabilidade, endividamento e o tamanho das empresas e Eegee. A Tabela 5 retoma as hipóteses da pesquisa e como os resultados obtidos se relacionam com elas.

Tabela 5 - Síntese das hipóteses e resultados obtidos

\begin{tabular}{ccccc}
\hline Hipóteses & \multicolumn{1}{c}{ Descrição } & $\begin{array}{c}\text { Relação } \\
\text { Esperada }\end{array}$ & $\begin{array}{c}\text { Relação } \\
\text { Observada }\end{array}$ & Decisão \\
$\mathrm{H}_{1}$ & $\begin{array}{c}\text { Percentual de ações nas mãos de diretores } \\
\text { executivos } \rightarrow \text { Não favorece a redução de } \\
\text { emissões de GEE }\end{array}$ & + & $\begin{array}{c}\text { Não } \\
\text { Significante }\end{array}$ & Não suportada \\
$\mathrm{H}_{2}$ & $\begin{array}{c}\text { Percentual de ações nas mãos de investidores } \\
\text { institucionais } \rightarrow \text { Favorece redução de emissões } \\
\text { de GEE }\end{array}$ & - & - & Suportada \\
$\mathrm{H}_{3}$ & $\begin{array}{c}\text { Percentual de ações nas mãos de investidores } \\
\text { ativistas } \rightarrow \text { Favorece redução de emissões de } \\
\text { GEE }\end{array}$ & - & Não significante & Não suportada \\
\hline
\end{tabular}

Fonte: Elaborada pelos autores

Revista de Gestão Social e Ambiental - RGSA, São Paulo, v. 14, n. 3, p. 93-109, set./dez. 2020. 
Desta forma, apresentam-se evidências estatísticas que dão suporte a hipótese 2 desta pesquisa, ou seja, um maior percentual de ações nas mãos de investidores institucionais favorece decisões que reduzem as emissões de GEE. As hipóteses 1 e 3 não foram suportadas neste estudo. Verificou-se relações não significantes envolvendo um maior percentual de ações nas mãos de diretores executivos e de investidores ativistas na eficiência na redução de emissões de GEE.

\section{DISCUSSÃO}

O estudo utiliza a teoria da agência para explicar a relação de determinadas estruturas de propriedade em decisões que favorecem (ou não) a redução de emissões de GEE. As empresas brasileiras, objeto deste estudo, são caracterizadas por uma estrutura de propriedade concentrada e a tomada de decisão dos gestores tem efeito no desempenho ambiental das empresas e nas metas de longo prazo. Os resultados da pesquisa evidenciam que empresas com um maior percentual de ações nas mãos de investidores institucionais tomam decisões que favorecem a redução de emissões de GEE. Por outro lado, empresas com um maior percentual de ações nas mãos de diretores executivos e investidores ativistas parecem não adotar medidas que induzam a mitigação de emissões de GEE. No geral, estes resultados estão alinhados com Abreu et al. (2021) que identificaram que as empresas subestimam ou compreendem mal os vários caminhos pelos quais as mudanças climáticas podem se manifestar na forma de impactos nos negócios. Os investidores não têm uma ideia clara até que ponto os governos podem fixar metas de emissões de GEE e nem do impacto dessas regulamentações no desempenho financeiro corporativo.

A confirmação da hipótese 2 é suportada pelos preceitos da teoria de agência de que propriedade institucional incentiva um maior compromisso das empresas com aspectos ambientais. A perspectiva de longo prazo dos investidores institucionais permite um maior engajamento em atividades que reduzem o impacto ambiental das operações das empresas. Além disso, assevera-se que a legitimação de atividades empresariais concede uma maior motivação para os investidores institucionais a implementarem projetos de mitigação de emissões de GEE. Os resultados encontrados corroboram os achados de Martínez-Ferrero e Lozano (2021) que analisaram a relação entre o nível de propriedade institucional e desempenho ambiental, social e de governança de 2474 empresas situadas em 16 países de economia emergente, os autores chegaram à conclusão de que quanto menor o nível de propriedade nas mãos de investidores institucionais, menor o desempenho ambiental, social e de governança e este efeito é atenuado à medida que a propriedade institucional atinja uma porcentagem significativa de 43\%. Elgergeni et al., (2018) investigaram o impacto da estrutura de propriedade na responsabilidade social corporativa de 300 mil empresas situadas no Reino Unido no período 2008-2012, os resultados mostram que um aumento nas participações institucionais promove um nível mais alto de engajamento voluntário em atividades sociais e ambientais. Resultados semelhantes foram identificados por Chen et al. (2020) que exploraram o relacionamento positivo e significante entre propriedade institucional e divulgação de aspectos sociais e ambientais, em 2000 empresas norte-americanas do índice Russell, no período de 2003 a 2006.

A relação não significante identificada entre a propriedade de ações nas mãos de diretores executivos e a redução das emissões de GEE reforça a ideia de que os custos associados aos investimentos ambientais superam os benefícios instrumentais e reduzem o interesse dos diretores executivos na alocação de recursos financeiros no desenvolvimento de estratégias climáticas. Adicionalmente, empresas que apresentam uma alta concentração de propriedade de diretores executivos estão sujeitas a uma menor pressão das partes interessadas na prestação de contas sobre o desempenho ambiental. Isso representa um dilema entre, por um lado, o desejo de um fluxo constante de pagamentos de dividendos e, por outro, a necessidade de implementar estratégias de baixo carbono (Abreu et al. 2021). Esses achados confirmam o estudo de Cai, Jo, \& Pan (2011) que examinaram 1946 empresas norte-americanas no período de 1996 a 2010 e observaram não existir

Revista de Gestão Social e Ambiental - RGSA, São Paulo, v. 14, n. 3, p. 93-109, set./dez. 2020. 
relação significante entre propriedade nas mãos de diretores executivos e atividades sociais e ambientais.

De modo semelhante, a relação não significante encontrada para os investidores ativistas e a redução de emissões de GEE pode ser explicada pelas características próprias do mercado acionário brasileiro, que é concentrado e tem uma baixa presença de investidores ativistas. Esses investidores que tentam exercer pressão nas empresas para evitar o excesso de poder do diretor executivo visando ganhos financeiros, parecem não conseguir mitigar o conflito de agência entre acionistas e gestores quando se trata de decisões sobre desempenho ambiental corporativo e em decisões sobre projetos de mitigação de emissões de GEE. Corroborando nossos achados, Walls et al. (2012) fizeram um estudo com 313 empresas norte-americanas no período entre 1997 e 2005 para estudar a relação entre governança corporativa e desempenho ambiental e concluíram que o ativismo dos acionistas não influencia positivamente no desempenho ambiental.

Em relação às variáveis de controle, a idade da empresa apresentou uma relação positiva e significante com o aumento de emissões de GEE, contrariando a ideia de que empresas com um tempo maior de atividade apresentam preocupações de longo prazo, e investem em práticas ambientais. Os estudos de Abban e Hasan (2020) e Ullah et al., (2019) explicam que empresas mais maduras, por estarem maior tempo em funcionamento e terem seu nicho de mercado, estão preocupadas com sua reputação e, portanto, tendem a divulgar mais informações de práticas ambientais e sociais. No caso brasileiro, uma possível explicação para os resultados encontrados pode ser que empresas mais maduras se acomodaram em relação aos investimentos ambientais ou não sofrem pressões das partes interessadas (Abreu et al., 2014)

\section{CONSIDERAÇÕES FINAIS}

Em face da complexidade das decisões sobre investimentos em projetos de mitigação de emissões de GEE, esse estudo amplia a compreensão dos fatores que influenciam as decisões dos gestores e investidores e suas interações com outras variáveis. Desta forma, ao analisar a associação entre determinadas estruturas de propriedade e a redução de emissões de GEE, a pesquisa oferece perspectivas para empresas, investidores e partes interessadas sobre a necessidade de estrutura regulatória estável que estabeleça claramente as expectativas com relação as emissões de GEE. Ressalta-se que, a partir do ano de 2019, houve retrocesso na política ambiental brasileira com medidas que enfraquecem a gestão ambiental e que tentam eliminar regulamentações existentes de combate ao meio ambiente e que geraram, como consequências, por exemplo, o aumento do desmatamento na Amazônia.

No campo teórico, esse trabalho agrega a discussão de estratégias climáticas e estrutura de propriedade no Brasil, um país que teve um aumento de $9,6 \%$ no ano de 2019 , em suas emissões de GEE, ficando distante de cumprir as suas metas definidas no Acordo de Paris (Seeg, 2020). Os resultados mostram que uma maior propriedade institucional favorece a adoção de estratégias climáticas por empresas brasileiras. Em relação às propriedades de ações em posse de diretores executivos e investidores ativistas, a pesquisa demonstra que estas estruturas não favorecem decisões que visam a implantação de estratégias climáticas. Adicionalmente, o estudo mostra que empresas brasileiras mais maduras não estão priorizando o desenvolvimento de atividades que levem à redução de emissões de GEE.

No que tange as limitações, esse estudo utilizou como amostra, empresas de uma única economia emergente, no caso o Brasil, caracterizado por ter uma estrutura de propriedade concentrada. Neste sentido, é possível ter resultados diferentes em outros países. Nota-se que poucas empresas realizam o inventário de emissões de gases do efeito estufa e menor ainda é o número daquelas que mensuram suas emissões indiretas nos escopos 2 e 3 , limitando-se apenas a emissões de escopo de emissões diretas (escopo 1), o que representa uma dificuldade na mensuração dos esforços para a redução das emissões de GEE. Embora se reconheçam as limitações empíricas do trabalho, estudos comparativos entre países podem melhorar o 
entendimento da influência de diferentes estruturas de propriedade na mitigação das emissões de GEE.

\section{REFERÊNCIAS}

Abban, A. R., \& Hasan, M. Z. (2020). The causality direction between environmental performance and financial performance in Australian mining companies - A panel data analysis. Resources Policy, 101894.

Abreu, M. C. S., Albuquerque, A. M. \& Freitas, A. R. P. (2014). Posicionamento estratégico em resposta às restrições regulatórias de emissões de gases do efeito estufa. Revista de Administração (FEA-USP), 49, 578-590.

Abreu, M. C. S., Webb, K., Araújo, F. S. M., \& Cavalcante, J. P. L. (2021). From "business as usual" to tackling climate change: Exploring factors affecting low-carbon decision-making in the canadian oil and gas sector. Energy Policy, 148, 111932.

Adebayo, T. S., Awosusi, A. A., Odugbesan, J. A., Akinsola, G. D., Wong, W. K., \& Rjoub, H. (2021). Sustainability of energy-induced growth nexus in Brazil: do carbon emissions and urbanization matter?. Sustainability, 13(8), 4371.

Arellano, M. (2003). Panel data econometrics. Oxford university press.

Autore, D. M., Clarke, N., \& Liu, B. (2019). Activist investors and open market share repurchases. Journal of Banking and Finance, 107, 105614.

Badrul, M., Arifur, M., Dessalegn, K., \& Mihret, G. (2018). The effect of board capital and ceo power on corporate social responsibility disclosures. Journal of Business Ethics, 150(1), 41-56

Braga, B., Hespanhol, I., Conejo, J. G. L., Mierzwa, J. C., de Barros, M. T. L., Spencer, M., Porto, M., Nucci, N., Juliano, N., \& Eiger, S. (2005). Introdução à engenharia ambiental: o desafio do desenvolvimento sustentável. Pearson Prentice Hall.

Bui, B., \& Fowler, C. J. (2019). Strategic responses to changing climate change policies: the role played by carbon accounting. Australian Accounting Review, 29(2), 360-375.

Bui, B., Houqe, M. N., \& Zaman, M. (2020). Climate governance effects on carbon disclosure and performance. British Accounting Review, 52(2), 100880.

Cadez, S., \& Czerny, A. (2016). Climate change mitigation strategies in carbon-intensive firms. Journal of Cleaner Production, 112, 4132-4143.

Cadez, S., Czerny, A., \& Letmathe, P. (2019). Stakeholder pressures and corporate climate change mitigation strategies. Business Strategy and the Environment, 28(1), 1-14.

Cai, Y., Jo, H., \& Pan, C. (2011). Vice or Virtue? The impact of corporate social responsibility on executive compensation. Journal of Business Ethics, 104(2), 159-173.

Chen, T., Dong, H., \& Lin, C. (2020). Institutional shareholders and corporate social responsibility. Journal of Financial Economics, 135(2), 483-504.

Cundill, G. J., Smart, P., \& Wilson, H. N. (2018). Non-financial shareholder activism: a process model for influencing corporate environmental and social performance. International Journal of Management Reviews, 20(2), 606-626.

Damert, M., \& Baumgartner, R. J. (2018). Intra-sectoral differences in climate change strategies: evidence from the global automotive industry. Business Strategy and the Environment, 27(3), 265281. 
Estrutura de propriedade e mitigação das emissões de gases do efeito estufa

Eleftheriadis, I., \& Anagnostopoulou, E. (2017). Measuring the level of corporate commitment regarding climate change strategies. International Journal of Climate Change Strategies and Management, 9(5), 626-644.

Elgergeni, S., Khan, N., \& Kakabadse, N. K. (2018). Firm ownership structure impact on corporate social responsibility: evidence from austerity U.K. International Journal of Sustainable Development and World Ecology, 25(7), 602-618.

Endo, K. (2020). Corporate governance beyond the shareholder-stakeholder dichotomy: Lessons from Japanese corporations' environmental performance. Business Strategy and the Environment, January, 1-9.

Frías-Aceituno, J. V, Rodríguez-Ariza, L., \& García-Sánchez, I. M. (2013). Is integrated reporting determined by a country' s legal system? An exploratory study. Journal of Cleaner Production, $44,45-55$.

Fuente, J. A., García-Sánchez, I. M., \& Lozano, M. B. (2017). The role of the board of directors in the adoption of GRI guidelines for the disclosure of CSR information. Journal of Cleaner Production, 141, 737-750.

García-Sánchez, I.-M. (2020). The moderating role of board monitoring power in the relationship between environmental conditions and corporate social responsibility. Business Ethics: A European Review, 29(1), 114-129.

Gasbarro, F., Iraldo, F., \& Daddi, T. (2017). The drivers of multinational enterprises' climate change strategies: A quantitative study on climate-related risks and opportunities. Journal of Cleaner Production, 160, 8-26.

Giovanni, L., \& Mauro, S. (2019). Towards a more ethical market: the impact of ESG rating on corporate financial performance. Social Responsibility Journal, 15(1), 11-27.

Goldstein, A., Turner, W. R., Gladstone, J., \& Hole, D. G. (2019). The private sector's climate change risk and adaptation blind spots. Nature Climate Change, 9(1), 18-25.

Goranova, M., Abouk, R., Nystrom, P. C., \& Soofi, E. S. (2017). Corporate governance antecedents to shareholder activism: a zero-inflated process. Strategic Management Journal, 38(2), 415-435.

Hadani, M., Doh, J. P., \& Schneider, M. (2019). Social movements and corporate political activity: Managerial responses to socially oriented shareholder activism. Journal of Business Research, 95, $156-170$.

Hadlock, C. J. (1998). Ownership, liquidity, and investment. The Rand Journal of Economics, 487508 .

Harjoto, M. A., \& Rossi, F. (2019). Religiosity, female directors, and corporate social responsibility for Italian listed companies. Journal of Business Research, 95, 338-346.

Howard-Grenville, J., Buckle, S. J., Hoskins, B. J., \& George, G. (2014). Climate change and management. Academy of Management Journal. 57 (2014), 615-623

Hossain, M., \& Farooque, O. (2019). The emission trading system, risk management committee and voluntary corporate response to climate change - a CDP study. International Journal of Accounting and Information Management, 27(2), 262-283.

Hua, G. W., Cheng, T. C. E., Zhang, Y., Zhang, J. L., \& Wang, S. Y. (2016). Carbon-constrained perishable inventory management with freshness-dependent demand. International Journal of Simulation Modelling (IJSIMM), 15(3).

Jahnke, P. (2019). Ownership concentration and institutional investors' governance through voice and exit. Business and Politics, 21(3), 327-350. 
Jackson, G., Bartosch, J., Avetisyan, E., Kinderman, D., \& Knudsen, J. S. (2020). Mandatory nonfinancial disclosure and its influence on CSR: an international comparison. Journal of Business Ethics, 162(2), 323-342.

Jamieson, D., \& Jacquet, J. (2020). The US response to covid-19 and climate change endangers the country and the world. One Earth, 2(6), 503-505.

Jaroenjitrkam, A., \& Zurbruegg, R. (2020). Does market power discipline CEO power ? An agency perspective. European Financial Management. 26(3), 724-752.

Ji, S.-H., Yoon, K.-C., Park, J., An, S.-B., \& Oh, H.-M. (2019). The relationship between CEO governance and social responsibility of service firms. Sustainability (Switzerland), 11(18).

Kennedy, P. (2003). A guide to econometrics. 5. th. Edition. Cambridge.

Khan, A., Muttakin, M. B., \& Siddiqui, J. (2013). Corporate governance and corporate social responsibility disclosures: evidence from an emerging economy. Journal of Business Ethics, 114(2), 207-223.

Kim, N., \& Kim, E. (2015). Board capital and exploration : from a resource provisional perspective. 63 (3), 2156-2174

Kumala, R., \& Siregar, S. V. (2020). Corporate social responsibility, family ownership and earnings management: the case of Indonesia. Social Responsibility Journal.

Lee, S. Y., Park, Y. S., \& Klassen, R. D. (2015). Market responses to firms' voluntary climate change information disclosure and carbon communication. Corporate Social Responsibility and Environmental Management, 22(1), 1-12.

Lopes de Sousa Jabbour, A. B., Vazquez-Brust, D., Chiappetta Jabbour, C. J., \& Andriani Ribeiro, D. (2020). The interplay between stakeholders, resources and capabilities in climate change strategy: converting barriers into cooperation. Business Strategy and the Environment. 29(3), 13621386.

Malik, F., Wang, F., Naseem, M. A., Ikram, A., \& Ali, S. (2020). Determinants of corporate social responsibility related to CEO attributes: an empirical study. SAGE Open, 28.

Martínez-Ferrero, J., \& Lozano, M. B. (2021). The nonlinear relation between institutional ownership and environmental, social and governance performance in emerging countries. Sustainability, 13(3), 1586.

Michelon, G., Rodrigue, M., \& Trevisan, E. (2020). The marketization of a social movement: Activists, shareholders and CSR disclosure. Accounting, Organizations and Society, 80, 101074.

Monks, R. A. G. (2008). Corpocracy: how CEOs and the business roundtable hijacked the world's greatest wealth machine--and how to get it back. John Wiley \& Sons.

Observatório do Clima (2020). Emissões do Brasil sobem 10\% no $1^{\circ}$ ano de Bolsonaro. Disponível em: https://www.oc.eco.br/emissoes-brasil-sobem-10-no-1o-ano-de-bolsonaro/. Acesso em: 10 out. 2020

Observatório do Clima (2021). "Passando a boiada": o segundo ano de desmonte ambiental sob Jair Bolsonaro. Recuperado em 10 out.2020, de: https://www.oc.eco.br/passando-a-boiada-osegundo-ano-de-desmonte-ambiental-sob-jair-bolsonaro/.

Oh, W.-Y., Cha, J., \& Chang, Y. K. (2017). Does ownership structure matter? The effects of insider and institutional ownership on corporate social responsibility. Journal of Business Ethics, 146(1), $111-124$. 
Estrutura de propriedade e mitigação das emissões de gases do efeito estufa

Oh, W. Y., Chang, Y. K., \& Martynov, A. (2011). The effect of ownership structure on corporate social responsibility: empirical evidence from Korea. Journal of Business Ethics, 104(2), 283-297.

Oikonomou, I., Yin, C., \& Zhao, L. (2020). Investment horizon and corporate social performance: the virtuous circle of long-term institutional ownership and responsible firm conduct. The European Journal of Finance, 26(1), 14-40.

Prasad, M., \& Mishra, T. (2017). Low-carbon growth for Indian iron and steel sector: exploring the role ofvoluntary environmental compliance. Energy Policy, 100(June 2016), 41-50.

Qi, L., Wang, L., \& Li, W. (2020). Do mutual fund networks affect corporate social responsibility? Evidence from China. Corporate Social Responsibility and Environmental Management, 27(2), 1040-1050.

Qian, W., Kaur, A., \& Schaltegger, S. (2018). Managing eco-efficiency development for sustainability: an investigation of top carbon polluters in Australia. In Accounting for Sustainability: Asia Pacific Perspectives, 103-124. Springer.

Qian, W., \& Schaltegger, S. (2017). Revisiting carbon disclosure and performance: legitimacy and management views. British Accounting Review, 49(4), 365-379.

Reid, E. M., \& Toffel, M. W. (2009). Responding to public and private politics: corporate disclosure of climate change strategies. Strategic Management Journal, 30(11), 1157-1178.

Sakawa, H., \& Watanabel, N. (2019). IPO underpricing and ownership monitoring in Japan. Asian Business and Management.

Shi, W., Connelly, B. L., Hoskisson, R. E., \& Ketchen, D. J. (2019). Portfolio spillover of institutional investor activism: an awareness-motivation-capability perspective. Academy of Management Journal.

Talbot, D., \& Boiral, O. (2015). Strategies for climate change and impression management: a case study among canada's large industrial emitters. Journal of Business Ethics, 132(2), 329-346.

Trinks, A., Mulder, M., \& Scholtens, B. (2017). Greenhouse gas emissions intensity and the cost of capital. SOM Research Reports, 17017-EEF.

Ullah, M. S., Muttakin, M. B., \& Khan, A. (2019). Corporate governance and corporate social responsibility disclosures in insurance companies. International Journal of Accounting and Information Management, 27(2), 284-300.

United Nations Environment Programme. (2019). Emissions Gap Report 2019.

Uysal, N., \& Tsetsura, K. (2015). Corporate governance on stakeholder issues: shareholder activism as a guiding force. Journal of Public Affairs, 15(2), 210-219.

Vitolla, F., Raimo, N., \& Rubino, M. (2020). Board characteristics and integrated reporting quality: an agency theory perspective. Corporate Social Responsibility and Environmental Management, $27(2), 1152-1163$.

Walls, J. L., Berrone, P., \& Phan, P. H. (2012). Corporate governance and environmental performance: Is there really a link? Strategic Management Journal, 33(8), 885-913.

Wei, J. (2020). Environmental, social, and governance proposals and shareholder activism. The Journal of Portfolio Management, 46(3), 49-64.

Wooldridge, M. (2009). An introduction to multiagent systems. John Wiley \& Sons.

Zou, H., Xie, X., Meng, X., \& Yang, M. (2019). The diffusion of corporate social responsibility through social network ties: from the perspective of strategic imitation. Corporate Social Responsibility and Environmental Management, 26(1), 186-198.

Revista de Gestão Social e Ambiental - RGSA, São Paulo, v. 14, n. 3, p. 93-109, set./dez. 2020. 
Victor D. Vasconcelos, Mônica C. Sá de Abreu, Vicente Lima Crisóstomo, Robson Silva Soe Rocha

RGSA adota a Licença de Atribuição CC BY do Creative Commons (https://creativecommons.org/licenses/by/4.0/). 